\section{Adherence to NICE guidelines for new glaucoma referrals}

Eye (2013) 27, 1230; doi:10.1038/eye.2013.188

Correction to: Eye (2013) 27, 571-572; doi:10.1038/eye.2013.2; published online 22 February 2013
R Chaudhary, N Dhillon and L Jones

Since the publication of the above article, the authors have noted that the name of the second author was incorrect. The correct details are given above.

The authors would like to apologise for this error. 